\title{
X-RAY ABSORPTION-DIFFRACTION QUANTITATIVE ANALYSIS USING PARALLEL BEAM XRD TECHNIQUE AND WD-XRF ELEMENTAL ANALYSIS RESULT FOR ORGANIC MATERIAL
}

\author{
Akihiko Iwata \\ Rigaku Latin America Ltda., São Paulo, SP, Brasil \\ iwata@rigaku.co.jp
}

\begin{abstract}
WD-XRF technique using the fundamental parameter method has been a mature multi-elemental analysis technique for various material, since it does not need any standard samples for the quantitative analysis. And recent developments of the synthesized multilayer mirror enable us to analyze light elements such as beryllium, boron, carbon and so on, and make it easy to obtain elemental compositions of organic compounds consisted from such light elements. XRD quantitative analysis has not been used widely compared to XRF analysis. Especially organic compounds tend to have preferred orientation by sample preparation process for XRD analysis, it obstructs the spread of XRD quantitative analysis. We carried out the X-ray absorption-diffraction quantitative analysis of organic material, acetylsalicylic acid, using mass absorption coefficients calculated from WD-XRF analysis results including light elements with an ordinary X-ray diffractometer which equipped parallel beam optics using a synthesized multi-layer mirror commonly used recently to eliminate the preferred orientation effects. The improved quantitative analysis result was observed.
\end{abstract}

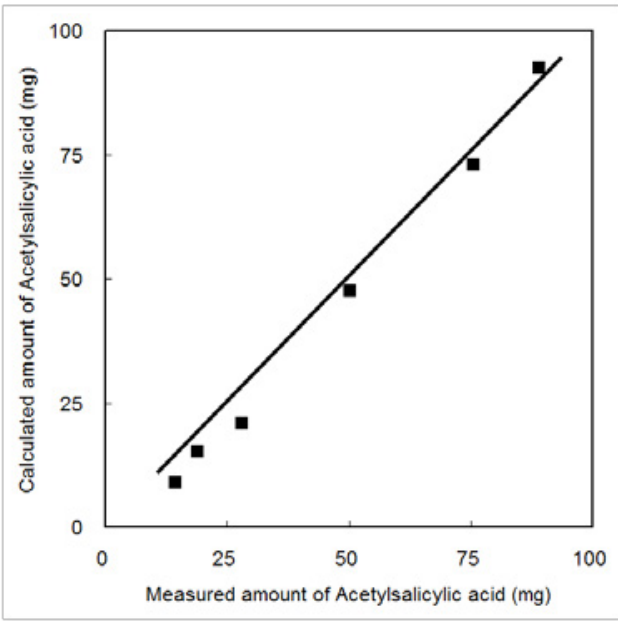

Fig.1 Measured amount of Acetylsalicylic acid vs. Calculated amount of Acetylsalicylic acid. 